\title{
Hormone
} Research

\section{ACTH 81}

Adrenocorticotropic hormone 9

ANF 116

Body composition 16

Breast cancer 42

- neoplasms 34

Cancer risk 34, 141

Cardiovascular disease 141

Catabolic stage 21

Chronic illness 95

Colorectal neoplasms 34

Compliance 104, 109

Constitutional delay of puberty 95

Cortisol 81, 121

Cost of GH 75

Criteria of GH treatment 75

Critical illness 21

Cushing's disease 81

- syndrome 81

Delayed puberty 95, 101

Diagnosis 81, 141

Diagnostic 75

Dose of GH 75

\section{Energy 55}

Family members 1

Final height 104, 127

Fluid retention 116

Gender 55

Gestation 55

GH deficiency 75,141

Gonadotropin releasing hormone agonist 64
Growth 64

- hormone 9, 42, 116, 121, 141

- - deficiency 70, 109, 127

- -, dosing, treatment design 70

- - peptide 16

- - releasing hormone 21

- _ - peptide 21

- _ - peptide-6 29

- - secretagogue(s) 9, 16,21,29

- - - receptor 1

- - - - ligands 1

- -, therapeutic, diagnostic 29

- _ therapy 70

- - treatment 109, 127

- _ compliance 113

Heart 9, 16

History 149

Hypogonadism 101

Hypogonadotropic 101

Hypothalamo-pituitary-gonadal axis 52

Hypothalamus 9

Insulin-like growth factor 34, 42, 70, 149

- _ - binding protein(s) 21, 42

- _ _ - protein-3 34

- - factor-I 21, 121, 141

Intrauterine growth retardation 109

Iodothyronines 121

Late puberty 95

Leptin 55

- receptors 55

Linear growth 16

Liquid growth hormone 109, 113

Lung cancer 42
Malignancy 42

Methods in measuring body composition 132

Monitoring GH treatment 141

Multiplication stimulating activity 149

Neonatal life 55

Non-suppressible insulin-like activity 149

ob Gene 55

Oestradiol 121

Organ system 16

Orphan receptor 1

Pituitary 9,21

Precocious puberty 64

Prediction algorithms 70

Prolactin 9

Prostate cancer 42

- neoplasms 34

Pubertal stage 55

Puberty 52, 55, 95, 101

Regulatory system 75

Renin-angiotensin-aldosterone system 116

Reproduction 55

Review 149

rhGH 132

Somatomedin 149

Somatotropin 104

Stress response 21

Sulphation factor 149

Triptorelin 64

Turner's syndrome 127

Variation 141 